11. In the American Journal of Mathematics, volume 34 (1912), page 173, Mr. Schweitzer has shown how to generate a quasi-four-dimensional geometry ${ }^{4} R_{4}^{(0)}$ by adjoining a point tactically to the system ${ }^{3} R_{3}$ and assuming the axiom " $\alpha R \beta \gamma \delta \in$ implies $\delta R \epsilon \alpha \beta \gamma$ " which ensures that the generating relation is alternating. The resulting system is sufficient for the usual threedimensional projective geometry if an axiom expressing Dedekind continuity (suitably modified for projective geometry) is added. This geometry ${ }^{4} R_{4}^{(0)}$ may be regarded as underlying a system of four-dimensional simplexes inscribed in a hypersphere. In the Archiv der Mathematik und Physik, volume 21 (1913), page 204, E. Study has remarked that the figure of five ordered real points, no four of which are coplanar, has a (single) property, "signatur" $(+)$ or $(-)$, which is not disturbed by positive real collineations. It seems simpler and altogether more convenient to regard Study's figure of five points with positive or negative "signatur" as a sensed simplex in quasifour space as indicated above.

$$
\text { O. D. KellogG, }
$$

Secretary of the Section.

\title{
THE INFINITE REGIONS OF VARIOUS GEOMETRIES.
}

BY PROFESSOR MAXIME BÔCHER.

(Read before the American Mathematical Society, September 8, 1913.)

Most geometers are now conscious that the introduction of points at infinity in such a way that in plane geometry they form a line, in three-dimensional geometry a plane, is, to a large extent, an arbitrary convention; but few of them would probably admit that this remark has much practical importance (except in so far as they might regard any question concerning the logical foundation of geometry as having practical importance) since the convention here referred to is commonly regarded as being the only desirable one. It is the object of the present paper to point out more explicitly and in greater detail than has, to my knowledge, 
been done before that this view is by no means justified.* For investigations in the realm of projective geometry, the conception of the line or the plane at infinity is the natural and appropriate one; and inasmuch as every geometric investigation can be forced into the projective mould, this conception of the infinite region can always be made to do duty. It is, however, not the most desirable one in all cases.

For the sake of simplicity I shall consider first merely plane geometry, and, indeed, the geometry of the real plane.

\section{\$1. The Projective Plane.}

In projective geometry we have to deal with the 8-parameter group of point transformations

$$
\begin{gathered}
X^{\prime}=\frac{a_{1} X+b_{1} Y+c_{1}}{a_{3} X+b_{3} Y+c_{3}} \\
Y^{\prime}=\frac{a_{2} X+b_{2} Y+c_{2}}{a_{3} X+b_{3} Y+c_{3}}
\end{gathered} \quad\left|\begin{array}{lll}
a_{1} & b_{1} & c_{1} \\
a_{2} & b_{2} & c_{2} \\
a_{3} & b_{3} & c_{3}
\end{array}\right| \neq 0,
$$

where $X, Y$ stand for non-homogeneous cartesian coordinates, which we will suppose to be rectangular. Every transformation of this group carries over points, in general, into points and collinear points into collinear points. Strictly speaking, the points on the line

$$
a_{3} X+b_{3} Y+c_{3}=0
$$

are not transformed at all; but the nearer one comes to such a point the farther away will the transformed point lie, and we consequently speak of these points as being "thrown to infinity," and thus introduce the conception of points at infinity in projective geometry. All these points at infinity, or ideal points, we regard as lying on a single line in order not to have any exception to the statement that collinear points go over into collinear points. We then introduce

* This article was already in type before I saw a paper, by H. Beck, Archiv d. Math. u. Physik, ser. 3, vol. 18, p. 43, which has much the same tendency. In that paper only the space of inversion is considered, and the questions taken up are in the main different from those here treated. The author seems not to have noticed that in my book of 1894 (cited below) I had explicitly described the infinite region for the complex plane of inversion, and implicitly (by the constant use of the term "Null sphere at infinity" with its equation) for spaces of higher dimensions. 
homogeneous coordinates $(x: y: t)$ by means of the equations

$$
X=\frac{x}{t}, \quad Y=\frac{y}{t},
$$

in order to be able conveniently to designate the various points at infinity. All this is so well known that the foregoing brief sketch will suffice.

If we now approach other groups of transformations with an open mind and in the same spirit in which we just approached the group (1), we shall be led in the same way to other geometries worthy of being considered side by side with projective geometry,* and each of these geometries will have just as much right to its own peculiar infinite region as has projective geometry.

\section{$\S 2 . \quad$ The Plane of Analysis. $\dagger$}

We shall consider in this section the geometry which is based on the 6-parameter group

$$
\begin{gathered}
X^{\prime}=\frac{\alpha_{1} X+\beta_{1}}{\gamma_{1} X+\delta_{1}} \quad\left|\begin{array}{ll}
\alpha_{1} & \beta_{1} \\
\gamma_{1} & \delta_{1}
\end{array}\right| \neq 0, \\
Y^{\prime}=\frac{\alpha_{2} Y+\beta_{2}}{\gamma_{2} Y+\delta_{2}} \quad\left|\begin{array}{cc}
\alpha_{2} & \beta_{2} \\
\gamma_{2} & \delta_{2}
\end{array}\right| \neq 0,
\end{gathered}
$$

where again, for the sake of concreteness, we assume all the quantities involved to be real.

Suppose, first, that neither $\gamma_{1}$ nor $\gamma_{2}$ is zero. Then (2) gives a transformation of the finite plane which carries over every point into a new position except that the points on the lines

$$
\gamma_{1} X+\delta_{1}=0, \quad \gamma_{2} Y+\delta_{2}=0
$$

are not transformed. If we take any point $\left(X_{1}, Y_{1}\right)$ on one of these lines, and allow another point $\left(X_{2}, Y_{2}\right)$ to approach it in such a way as never to lie on the line in question, then the image of this second point recedes to infinity. In this sense we may say that the lines (3), (4) are both thrown to infinity.

* Cf. Klein's Erlanger Programm (1872), where, however, the point with which we are here concerned, the character of the infinite region, is not brought out.

† Cf. Osgood, Transactions, vol. 13 (1912), p. 159. 
Similarly, since the inverse of (2) is

$$
X=\frac{-\delta_{1} X^{\prime}+\beta_{1}}{\gamma_{1} X^{\prime}-\alpha_{1}}, \quad Y=\frac{-\delta_{2} Y^{\prime}+\beta_{2}}{\gamma_{2} Y^{\prime}-\alpha_{2}},
$$

we see that there are also two lines

$$
\gamma_{1} X^{\prime}-\alpha_{1}=0, \quad \gamma_{2} Y^{\prime}-\alpha_{2}=0
$$

which come from infinity.

If one but not both of the quantities $\gamma_{1}, \gamma_{2}$ are zero, only one of the lines (3), (4) will go to infinity (the other equation no longer representing any locus), and only one of the lines (5), (6) will come from infinity; while if $\gamma_{1}=\gamma_{2}=0$, there are no exceptional lines of this sort.

Apart from these exceptional lines, every line $x=$ const., and also every line $y=$ const., goes over into another line of the same form; and every line of one of these forms comes from another such line. These lines we shall find play a fundamental rôle in the kind of geometry we are now considering, and it will be convenient to designate them as ground-lines, distinguishing between two kinds of groundlines according as the equation is $x=$ const. or $y=$ const. We may then say

The transformations (2) are one-to-one point transformations which carry every ground-line into a ground-line of the same kind.

This statement, however, is at present not accurate since it admits certain exceptions. It becomes accurate only when, as we shall now do, we introduce points at infinity (ideal points) in such a way that they form two new ideal groundlines, one of each kind. This may be easily effected by synthetic methods. We will, however, proceed analytically by the use of homogeneous coordinates defined by the equations

$$
X=\frac{x_{1}}{x_{2}}, \quad Y=\frac{y_{1}}{y_{2}} .
$$

These, it should be noticed, are not the homogeneous coordinates of projective geometry, a point being determined here by four coordinates $\left(x_{1}: x_{2}, y_{1}: y_{2}\right)$ involving, however, only two ratios. The finite points are those for which neither $x_{2}$ nor $y_{2}$ is zero, while the points at infinity are, by definition, sets of coordinates in which either $x_{2}$ or $y_{2}$ or both are zero, 
but where not both the $x$ 's (and also not both the $y$ 's) are zero. These last mentioned sets of coordinates we do not speak of as being points at all. Moreover we agree, that two points at infinity shall be identical when and only when the $x$ 's of one are proportional to the $x$ 's of the other, and also the $y$ 's of one are proportional (though perhaps with a different factor of proportionality) to the $y$ 's of the other. This convention is the natural one to make, since finite points will be identical under, and only under, the same conditions.

Every homogeneous equation of the first degree in $y_{1}, y_{2}$ represents a line parallel to the axis of $x$ (an $x$-ground-line) except the equation $y_{2}=0$ which is satisfied only by ideal points and which we speak of as the $x$-ground-line at infinity.

Similarly the equation $x_{2}=0$ gives us the $y$-ground-line at infinity.

These two ideal ground-lines obviously contain all points at infinity. They have one and only one point in common, namely $(1: 0,1: 0)$, which we speak of as the double point at infinity in distinction to the infinite number of simple points at infinity where only one of the two quantities $x_{2}, y_{2}$ is zero.

The transformation (2) may be written

$$
\begin{array}{lll}
\rho x_{1}{ }^{\prime}=\alpha_{1} x_{1}+\beta_{1} x_{2}, & \sigma y_{1}{ }^{\prime}=\alpha_{2} y_{1}+\beta_{2} y_{2}, & \alpha_{1} \delta_{1}-\beta_{1} \gamma_{1} \neq 0, \\
\rho x_{2}{ }^{\prime}=\gamma_{1} x_{1}+\delta_{1} x_{2}, & \sigma y_{2}{ }^{\prime}=\gamma_{2} y_{1}+\delta_{2} y_{2}, & \alpha_{2} \delta_{2}-\beta_{2} \gamma_{2} \neq 0 .
\end{array}
$$

We pass now to straight lines other than ground-lines. Such a line may be written

$$
A X+B Y+C=0,
$$

where neither $A$ nor $B$ is zero; or, in homogeneous coordinates,

$$
A x_{1} y_{2}+B y_{1} x_{2}+C x_{2} y_{2}=0 .
$$

This is a special case of the general bilinear equation

$$
a x_{1} y_{1}+b x_{1} y_{2}+c x_{2} y_{1}+d x_{2} y_{2}=0 .
$$

This equation is readily seen to represent, in general, an equilateral hyperbola with ground-lines as asymptotes. It may, however, as we have just seen represent a single straight line, not a ground-line, or it may represent any pair of ground- 
lines, one of each set. All these curves we shall speak of as bilinear curves; the last case, which is characterized algebraically by the vanishing of the determinant* $a d-b c$, being designated as a singular bilinear curve. A non-singular bilinear curve is a straight line or a hyperbola according as it does or does not pass through the double point at infinity. It should be noticed, however, that a single ground-line is not a bilinear curve, but is something simpler.

It is clear that all transformations (7) carry bilinear curves into bilinear curves leaving them singular or non-singular as the case may be. All non-singular (and also all singular) bilinear curves are equivalent under the group (7).

It will thus be seen that in this kind of geometry the classification of curves as straight lines, conics, etc., is an artificial one; some straight lines being, for instance, ground-lines and some bilinear curves. This fact becomes still clearer if we consider some of the fundamental properties of the various curves.

Two distinct ground-lines of the same kind have no point in common. For two such $y$-ground-lines are given by the equations

$$
\begin{aligned}
& p_{1} x_{1}+q_{1} x_{2}=0 \\
& p_{2} x_{1}+q_{2} x_{2}=0
\end{aligned} \quad p_{1} q_{2}-p_{2} q_{1} \neq 0,
$$

which are satisfied only by $x_{1}=x_{2}=0$. This we do not speak of as a point.

Two ground-lines of different kinds have one and only one point in common. In particular, two different finite, $x$-groundlines have different points at infinity, namely the points where they meet the $y$-ground-line at infinity. It will not do in this case to say that parallel lines meet at infinity.

A ground-line and a non-singular bilinear curve always have one and only one point in common. A general algebraic proof may readily be given, or we may first reduce the ground-line (which we may suppose to be a $y$-line) and the bilinear curve to the normal forms

$$
x_{1}=0, \quad x_{1} y_{1}+x_{2} y_{2}=0
$$

by a transformation (7), as is clearly always possible. These

${ }^{*}$ We note in passing that this determinant is an invariant (combinant) of the bilinear curve under the transformation (7). 
curves have obviously the point $(0: 1,1: 0)$, and no other point, in common.

Two distinct non-singular bilinear curves have not more than two points in common. If there is any point of intersection, let $P$ be such a point, and throw it to the double point at infinity by a transformation (7). Then the bilinear curves become straight lines, and these can intersect at most in one point besides the double point at infinity.

It is readily seen from this proof, or otherwise, that the case of a single point of intersection may be regarded as the limit of cases in which the curves intersect in two points and where these two points approach each other. We say, then, in this case that the curves are tangent to each other. It is readily seen that this definition of contact coincides with the ordinary one when the point of contact is finite. Contact at infinity, on the other hand, means something very different here and in projective geometry. This is illustrated by the following facts:

A ground-line cannot touch a bilinear curve, since it always meets it in only one point. In particular, an equilateral hyperbola whose asymptotes are ground-lines must not now be regarded as being tangent to these ground-lines at infinity.

Two parallel straight lines, not ground-lines, must be regarded as non-singular bilinear curves which have contact at the double point at infinity.

Two bilinear curves (equilateral hyperbolas) with one asymptote in common do not in general have contact with one another at infinity. They do this, however, if they not only have a common asymptote but are of the same size without coinciding.

\section{§3. Correspondence of the Plane of Analysis to a Ruled Quadric.}

The two sets of ground-lines of the plane of analysis strongly suggest the two sets of rulings on a non-singular quadric surface. In each case every line of one set meets every line of the other set, but meets no other line of its own set. That this is not merely a superficial analogy may be seen as follows:

Let $S$ denote any unparted hyperboloid or hyperbolic paraboloid in projective space, and designate one of the two sets of rulings arbitrarily as the first set, the other as the second set. It is well known that the cross-ratio of the range 
in which four rulings of one set are cut by a ruling of the other set is independent of the choice of this ruling, and that this cross-ratio is called the cross-ratio of the four rulings. Now let us choose three fixed rulings of the first set and attach to every ruling of this set a value of the parameter $X$ equal to the cross-ratio which this ruling makes with the three fixed rulings. In this way every ruling of the first set is uniquely determined by a value of $X$, the three originally selected rulings corresponding to the values $X=0,1, \infty$. Similarly we determine the rulings of the second set by the values of a parameter $Y$. Since every point on $S$ is determined as the intersection of one ruling of the first with one of the second set, we have thus a system of coordinates $(X, Y)$ between whose real values and the real points on $S$, finite or at infinity, there is a continuous one-to-one correspondence, provided we include the values $X=\infty$ and $Y=\infty$. We thus establish a continuous one-to-one correspondence between the points of $S$ and the points of the plane of analysis of such a sort that to the two sets of rulings of $S$ correspond the two sets of ground-lines of the plane of analysis.* Moreover the transformations (2) of the plane of analysis evidently correspond to the transformations of $S$ into itself which carry over every ruling of each set into another ruling of the same set in such a way that each set of rulings is transformed projectively into itself. Such transformations are yielded by those collineations of space which carry over $S$ into itself without interchanging the two sets of rulings; and conversely it may be proved that every transformation of $S$ into itself, of the kind we want, may be obtained in this way. Thus we may say:

Abstractly considered, the real plane of analysis with its real transformations (2) is identical with a real, non-singular, ruled quadric $S$ and such of its real collineations into itself as do not interchange the rulings of the two sets. If, however, we wish to get an intuitional substratum for this abstract theory, the plane of analysis would seem to be the simpler from every point of view except that it is, at first, a little unfamiliar. $\dagger$

Several generalizations of what has just been said will at once suggest themselves.

* By taking for $S$ a hyperbolic paraboloid, we can, if we wish, make the infinite region of the plane of analysis correspond to the infinite region of $S$.

$\dagger$ The quadric surface $S$ also has its peculiar advantages owing to the way it is immersed in three-dimensional space. 
In the first place, no difficulty is introduced by allowing all the quantities considered to be complex. In this way we get the complex plane of analysis, on the one hand, and the complex surface $S$, on the other; that is, not merely the real points of $S$, which we have considered exclusively so far, but also its complex points. Moreover, there is no necessity now to assume that $S$ has real rulings, or even that it has real points: it may be any non-singular quadric.*

On the other hand, either in the real or in the complex plane of analysis, we may consider not the group of transformations (2), but the larger group consisting of these transformations (which we will now call the direct transformations) and also of the indirect transformations obtained by combining them with the transformation

$$
X^{\prime}=Y, \quad Y^{\prime}=X .
$$

This larger group obviously corresponds to the group of all the collineations of $S$ into itself. From this point of view we may say:

The geometry of analysis in the plane, and the projective geometry on any non-singular quadric surface, are abstractly identical.

\section{$\S 4$. The Plane of Inversion.}

This last theorem recalls one of Klein's early discoveries $\dagger$ namely that the geometry of inversion in the plane is abstractly identical with the projective geometry on any non-singular quadric surface. By combining these two facts we get the result:

The geometry of analysis in the plane and the geometry of inversion in the plane are abstractly identical.

This result, like the two from which we have deduced it, is true, however, only if we disregard all questions of reality. Let us, then, go back and consider the geometry of inversion for its own sake, at first in a very elementary manner.

We start from the real finite plane and its rectangular cartesian coordinates $(X, Y)$, and consider the 6-parameter group of transformations generated by all rigid motions

* We mention in passing that the bilinear curves may readily be shown to correspond to the plane sections of $S$, a fact which is, of course, equally true if we restrict ourselves to reals.

† Math. Annalen, vol. 5 (1872), p. 267. 
combined with inversions in circles and reflections in lines. Since an inversion throws the points near the center of the circle to a great distance, and brings the points at a great distance into the neighborhood of the center, we are in the habit of saying that it throws the center to infinity, and vice versa; and since, except for this, we have a one to one transformation, we are here led, in precisely the manner of $\S \S 1,2$, to introduce the conception of a single point at infinity, and to say: In the geometry of inversion of the real plane, the infinite region shall be regarded as forming a single point. This point of view is so familiar, through its importance in the theory of functions of a single complex variable, that we need not insist on it further. It is, indeed, the one case in which it is generally conceded, though often grudgingly, that the conception of the line at infinity is not the only possible one. We recall that the simplest class of curves here is that which consists of all straight lines and all circles (we consider here only real loci), these curves being carried into one another by the transformations of the group. Straight lines differ from circles merely in that they pass through the point at infinity. Two straight lines intersect in general in two points, one of which is at infinity; while if the lines are parallel they must be regarded as tangent to each other at infinity. Etc.

Far less commonly recognized is what we get when we consider the geometry of inversion in the complex plane.* We must here consider the two sets of minimal lines

$$
X+\sqrt{-1} Y=\text { const., } X-\sqrt{-1} Y=\text { const. }
$$

It is readily seen that every motion of the plane in itself carries over a minimal line into a minimal line of the same set, while every reflection in a line $\dagger$ and every inversion in a circle $\ddagger$ carries over a minimal line into a minimal line of the other set. In the case of inversions, however, there are two exceptions: points on the minimal lines through the center of the circle of inversion are not transformed at all, but may,

*Cf. Study: "Das Apollonische Problem," Math. Annalen, vol. 49 (1897), p. 497, and E. v. Weber: "Zur Theorie der Kreisverwandtschaften in der Ebene," Bayerische Sitzungsberichte, vol. 31 (1901), p. 367, in neither of which long treatments is a word said concerning the nature of the infinite region.

$\dagger$ Reflection is defined when and only when the line is not a minimal line.

$\neq$ Inversion in a circle is defined when and only when the circle is not a null circle. 
as in previous cases, be said to be thrown to infinity. Thus we are led to expect that in the geometry of inversion of the complex plane the infinite region should be taken as consisting of one real ideal point (the point at infinity, of the real plane) and through it two ideal minimal lines containing no other real point.*

All this can be put on a firm basis by the use of circular coordinates

$$
\Xi=X+\sqrt{-1} Y, \quad \mathrm{H}=X-\sqrt{-1} Y .
$$

In terms of these coordinates the group of direct circular transformations, that is, those which do not interchange the two sets of minimal lines, may be written $\dagger$

$$
\begin{array}{ll}
\Xi^{\prime}=\frac{\alpha_{1} \Xi+\beta_{1}}{\gamma_{1} \Xi+\delta_{1}} & \left|\begin{array}{ll}
\alpha_{1} & \beta_{1} \\
\gamma_{1} & \delta_{1}
\end{array}\right| \neq 0, \\
\mathrm{H}^{\prime}=\frac{\alpha_{2} \mathrm{H}+\beta_{2}}{\gamma_{2} \mathrm{H}+\delta_{2}} & \left|\begin{array}{cc}
\alpha_{2} & \beta_{2} \\
\gamma_{2} & \delta_{2}
\end{array}\right| \neq 0 .
\end{array}
$$

We may also introduce homogeneous circular coordinates $\left(\xi_{1}: \xi_{2}, \eta_{1}: \eta_{2}\right)$ by means of the relations

$$
\Xi=\frac{\xi_{1}}{\xi_{2}}, \quad \mathrm{H}=\frac{\eta_{1}}{\eta_{2}},
$$

when the 6-parameter group of direct circular transformations becomes

$$
\begin{array}{ll}
\rho \xi_{1}{ }^{\prime}=\alpha_{1} \xi_{1}+\beta_{1} \xi_{2}, & \sigma \eta_{1}{ }^{\prime}=\alpha_{2} \eta_{1}+\beta_{2} \eta_{2}, \\
\rho \xi_{2}{ }^{\prime}=\gamma_{1} \xi_{1}+\delta_{1} \xi_{2}, & \sigma \eta_{2}{ }^{\prime}=\gamma_{2} \eta_{1}+\delta_{2} \eta_{2} .
\end{array}
$$

The indirect circular transformations come by combining these direct transformations with an interchange of the $\xi$ 's with the $\eta$ 's.

It will be seen that the formulæ just written are identical with those used in $\S 2$, except that $\Xi$ and $\mathrm{H}$ now take the place of $X$ and $Y$, and that, for real points, $\Xi$ and $H$ are complex. Hence, disregarding questions of reality, the

* Cf. my book, Über die Reihenentwicklungen der Potentialtheorie, Leipzig, Teubner, 1894, p. 27, footnote. The whole treatment there given was written under the inspiration of Klein.

† Cf. E. v. Weber, loc. cit., p. 382 . 
general theorem at the beginning of this section follows again, this time without reference to quadric surfaces.

A systematic presentation of the geometry of inversion from the point of view here explained, namely that the infinite region consists of two minimal lines intersecting in a real point, would be most desirable. In it, two distinct circles intersect in only two points, the circular points at infinity of projective geometry having now no existence. Every circle, to be sure, has two ideal points, namely the imaginary points where it cuts the ideal minimal lines; but these points are in general different for different circles. In the case of concentric circles, which have no finite points in common, these ideal points are the same for one circle as for the other. Two distinct circles always intersect in two and only two points, which may coincide, in which case we say the circles are tangent; and the same is true of a circle and a line (not minimal), or of two lines (not minimal); but a minimal line meets a circle or an ordinary line in only one point, so that there is no possibility of contact here.

As a final illustration of the desirability of this point of view I mention the subject of foci. According to the ordinary definition, a focus of a plane curve is a point of intersection of two minimal lines, one of each set, both of which are tangent to the curve. The scope of this definition is not, however, coextensive in the projective plane and in the plane of the geometry of inversion, since contact at infinity means something very different in the two cases. Thus, in the projective plane, the center of a circle is its focus, since the minimal lines through the center touch the circle at the circular points at infinity. In the plane of the geometry of inversion, a circle has no focus (any more than a straight line has) since, as we have just noted, a minimal line cannot touch it. Since in the plane of the geometry of inversion the 6-parameter group of circular transformations is without exception a contact transformation, and carries minimal lines into minimal lines, it is clear that the relation of a focus to a plane curve is always invariant with regard to circular transformations.

The reader should contrast the perfect simplicity and generality of such statements as we have just been making with the far less perfect form the geometry of inversion takes when we use the projective plane and therefore have the 
circular points at infinity as fundamental points of the transformations of the group.* In order to get the complete benefit of our point of view, a suitable terminology (which should be neither uncouth nor very extensive) should be introduced. In particular, some name should be given to the class of curves consisting of all circles, and all lines which are not minimal lines. If, following the analogy of $\S 2$, we call such curves bilinear curves and those which consist of a pair of minimal lines singular bilinear curves, we may say: any two distinct non-singular bilinear curves intersect in two points either distinct or coincident. Let the reader enunciate completely, and correctly the facts in the projective plane which correspond to this, and he will get some idea of the gain in simplicity to which we have referred. $\dagger$

\section{§5. Three-Dimensional Space.}

In one-dimensional geometry (the straight line) there is no distinction between the three kinds of spaces with which we have been dealing, the groups of transformations in the three cases being identical.

In two dimensions, we have seen that two of the three geometries differ only in questions of reality, while the third, projective geometry, is essentially different from them.

When we get above two dimensions, all three geometries are essentially different even without the introduction of questions of reality. For the sake of simplicity, we confine ourselves to three dimensions. Unless the contrary is stated, we suppose all quantities to be complex.

The three dimensional projective space with its plane at infinity is so familiar that a mere reference is sufficient. The group is, of course, the 15-parameter group of all non-singular collineations.

In the space of analysis we have the 9-parameter group

$$
X^{\prime}=\frac{\alpha_{1} X+\beta_{1}}{\gamma_{1} X+\delta_{1}}, \quad Y^{\prime}=\frac{\alpha_{2} Y+\beta_{2}}{\gamma_{2} Y+\delta_{2}}, \quad Z^{\prime}=\frac{\alpha_{3} Z+\beta_{3}}{\gamma_{3} Z+\delta_{3}},
$$

* Cf. the classical treatment given from this point of view by Darboux in his book, Sur une Classe remarquable de Courbes et de Surfaces algebriques, 1872 .

$\dagger$ We mention, in passing, that it is perfectly possible to study projective geometry in the plane of analysis or in the plane of the geometry of inversion. The collineations will then, in general, be quadratic transformations with the double point at infinity as a fundamental point and the ideal ground-lines (or minimal lines) as fundamental lines. 
where we suppose the determinant of each of these three linear functions to be different from zero. The planes

$$
X=\text { const., } Y=\text { const., } \quad Z=\text { const. }
$$

we speak of as the three sets of ground-planes; and the lines parallel to the coordinate axes, as the three sets of groundlines. We then introduce our ideal points, precisely as in $\$ 2$, so that they completely fill three ideal ground-planes; one belonging to each set. These ground-planes intersect in three ideal ground-lines, one of each set; and all three ideal groundplanes (and also all three of the ideal ground-lines just mentioned) have one and only one point in common, the triple point at infinity. Our nine-parameter group is then, without exception, a one-to-one continuous point transformation. It carries over every ground-line (or ground-plane) into another ground-line (or ground-plane) of the same set. The whole subject may be developed farther along precisely the lines of $\$ 2$.

Finally in the geometry of inversion we deal with the 10-parameter group of circular transformations generated by all motions, reflections in planes (not minimal), and inversions in spheres (not null). By entirely elementary computation we find that these generating transformations all carry over minimal lines into minimal lines, except that an inversion throws all the minimal lines through the center of the sphere of inversion to infinity. Moreover the points on these minimal lines are the only points which are not transformed. The minimal lines through a point form a minimal cone, and every minimal cone is carried into another minimal cone, with the exception just noted of the minimal cone with vertex at the center of inversion, which is thrown to infinity, and also with the exception of the minimal cones whose vertices are on the minimal cone just mentioned. Such a minimal cone becomes a minimal plane, and, conversely, every minimal plane becomes a minimal cone of the kind last mentioned. The generating transformations other than inversions of course carry over minimal planes into minimal planes.

Out of this complexity we can bring the most beautiful order by introducing ideal points of which one is real (the point at infinity of the real geometry of inversion) and all the others imaginary, and which fill completely a minimal cone with the real ideal point as vertex. A minimal cone being defined as the locus of all minimal lines through a given point, 
the minimal planes now appear merely as special cases of minimal cones, namely those whose vertices are imaginary ideal points. We may now say, without exception, that in the space of the geometry of inversion, as thus formed, the 10-parameter group of circular transformations is a one-to-one continuous transformation which carries over every minimal line into a minimal line. All this may be put on a firm foundation most conveniently by using as an analytic instrument Darboux's pentaspherical coordinates.

The simplest class of surfaces in this geometry (we will call them linear surfaces) consists of all planes and spheres. Among these we distinguish the singular surfaces, namely the null spheres (or minimal cones) of which, as we have seen, the minimal planes form a special case. Not only will linear surfaces go into linear surfaces (remaining singular if they were so originally) but any two non-singular (and also any two singular) linear surfaces are equivalent with regard to the 10-parameter group of circular transformations.

Here, again, the circle at infinity of projective geometry has no existence. Every sphere has ideal points, namely the points of the curve in which it meets the ideal minimal cone, and this curve may properly be called a circle since it is the intersection of two spheres, namely the given sphere and any sphere concentric with it. Two spheres which are not concentric will, however, not pass through the same ideal circle, as they do in projective space, but through different ones.

We recalled at the beginning of $\$ 4$ an important result of Klein. If we take as our quadric surface a real, non-singular quadric with real points but imaginary generators, or, in particular, a sphere, that result may, as is well known, be stated more precisely as follows:

When we consider questions of reality the geometry of inversion in the plane is abstractly identical with the projective geometry of a sphere with real non-vanishing radius and real center.

The import of this statement is not merely that there is a one-to-one correspondence between the points of the sphere and the points of the plane of the geometry of inversion of such a sort that real points correspond to real points, but also that there is a one-to-one correspondence between the circular transformations of the plane and the collineations of the spherical surface into itself. The most elementary way 
of establishing this correspondence is, of course, by means of a stereographic projection of the plane on the sphere. Now this stereographic projection may also be regarded as an inversion of space which carries over the plane into the sphere. We thus look at the sphere as lying not in projective space but in the space of the geometry of inversion, and we are thus led to inquire whether the transformations of the sphere into itself with which we are concerned may not also be obtainable by means of a subgroup of the 10-parameter group of circular transformations of space. A count of constants $(10-4=6)$ suggests that there should be a 6-parameter group of circular transformations of space which carry over the sphere into itself. A closer examination shows that this is actually the case, but that instead of there being a one-to-one correspondence between the circular transformations of the plane and the transformations of this subgroup, the correspondence is oneto-two; either of the two circular transformations of space which give the same transformations of the sphere into itself being obtainable from the other by following, or preceding, it by an inversion in the given sphere. Thus we get the geometry of inversion of the plane either by considering the 6-parameter group of collineations of projective space which carry over a real non-singular sphere into itself or the 6-parameter group of circular transformations of the space of inversions which carry over a real non-singular sphere (or a real plane) into itself.

If what has here been said does not persuade all readers of the desirability of using, on occasion, other spaces than projective space, it is hoped that it may at least make clear the desirability of stating explicitly in all cases what space it is that is being used. Papers dealing with transformations which throw points to infinity are not in a satisfactory form unless it is made clear what infinite region is assumed.

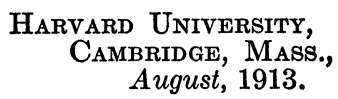

